\title{
Nasalidade no Aguaruna
}

\author{
Seung Hwa Lee \\ UNIVERSIDADE FEDERAL DE MINAS GERAIS
}

\section{Abstract}

In the previous studies of autosegmental phonology, the nasal harmony was considered as the universally conditioned harmony process, like tone, [ \pm back], [ATR]. In this paper, following the theory of Clements (1991), I propose that the language particular association convention should be assumed to explain the nasal harmony in Aguruna. 


\section{INTRODUÇÃO*}

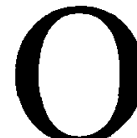

presente trabalho tem como objetivo explicar vários proces sos de harmonização nasal encontrados no Aguaruna, uma língua falada por, aproximadamente, 45 mil falantes, da região norte da Amazônia, numa área pertencente ao Peru. Esses fenômenos, registrados também em outras línguas indígenas da América Latina, já foram analisados por diversos autores e sob diferentes perspectivas teóricas (Piggott, 1992; Hulst \& Smith, 1982b; McCarthy, 1988).

O quadro teórico adotado na presente análise será o da Geometria de Traços, encontrada em Clements (1985, 1987a, 1987b, 1989, 1991) e conhecida na literatura como Fonologia Auto-segmental. De acordo com este modelo, o traço [nasal] é tratado no nível auto-segmental, de modo que a harmonização nasal do Aguaruna pode ser vista como um processo bastante simples.

Payne (1974) e Corbera (1980), adotando a linha da Fonologia Gerativa Padrão, explicam esses fenômenos no Aguaruna através de um grande número de regras complicadas e abstratas.

No que se refere a trabalhos realizados sobre nasalidade à luz do modelo da Fonologia Auto-segmental, destacam-se, entre outros, os de Hyman (1982), Hulst \& Smith (1982b), Cohn (1990), Piggott (1987, 1992) e Moon (1988). Para esses autores, a harmonização nasal é um processo sujeito somente a Condiçøes Universais, ou seja, esses autores não consideram que o processo de harmonização nasal seja sujeito a restriçס̃es de línguas particulares, tais como os elementos fonológicos [ATR], [ \pm back], o tom, etc. Piggott (1992), por exemplo, mostrou que o processo de harmonização nasal está sujeito a dois paradigmas possíveis.

O presente trabalho buscará mostrar que esșes dois paradigmas 
não são suficientes para explicar a harmonização nasal do Aguaruna, que é uma língua em que existem segmentos-alvo e segmentos opacos mas não existem segmentos transparentes. Para explicar a harmonização nasal, nessa língua, será levada em conta a Restrição de Boa Formação (Well-formedness Condition, doravante WFC), assumindo que a harmonização nasal do Aguaruna não está sujeita a WFC usada na análise do traço [ATR] por Halle \& Vergnaud (1981), na análise do tom por Goldsmith (1976) e Halle \& Vergnaud (1982) e na análise do processo de nasalização por Piggott (1992). ${ }^{1}$ A harmonização nasal do Aguaruna será tratada como determinada por uma WFC restrita e por uma Convenção de Associação. Serão, portanto, apresentados argumentos em favor da necessidade de se restringir a noção de WFC. Em outras palavras, neste trabalho, argumenta-se em favor da idéia de que o processo de harmonização nasal é diferente dos processos de harmonização de [ATR] e de tom e, por isso, o traço [nasal] deve ser tratado não apenas pelas condições universais, mas, também, por restrições da língua particular.

\section{INVENTÁRIO DE FONEMAS E REPRESENTAÇĀO DE NASALIDADE}

Os fonemas do Aguaruna são os apresentados em (1):2

(1)

a. Consoantes ${ }^{3}$

\begin{tabular}{|l|c|c|c|c|c|}
\hline & bilabial & alveolar & palato-alveolar & velar & glotal \\
\hline oclu. sur. & $\mathrm{p}$ & $\mathrm{t}$ & & $\mathrm{k}$ & $?$ \\
\hline oclu. son. & $\mathrm{b}$ & $\mathrm{d}$ & & & \\
\hline africadas & & $\mathrm{c}$ & $\mathrm{c}$ & & \\
\hline fricativas & & $\mathrm{s}$ & $\mathrm{s}$ & $\gamma$ & \\
\hline nasais & $\mathrm{m}$ & $\mathrm{n}$ & & $\mathrm{n}$ & \\
\hline glides & $\mathrm{w}$ & & $\mathrm{y}$ & & $\mathrm{h}$ \\
\hline
\end{tabular}


b. Vogais

\begin{tabular}{|l|c|c|c|}
\hline & anterior & central & posterior \\
\hline alta & $\mathrm{i}$ & $\dot{\mathrm{t}}$ & $\mathrm{u}$ \\
\hline baixa & & $\mathrm{a}$ & \\
\hline
\end{tabular}

Em relação à nasalidade, os fonemas do Aguaruna podem ser representados das seguintes formas (cf. Clements, 1991):

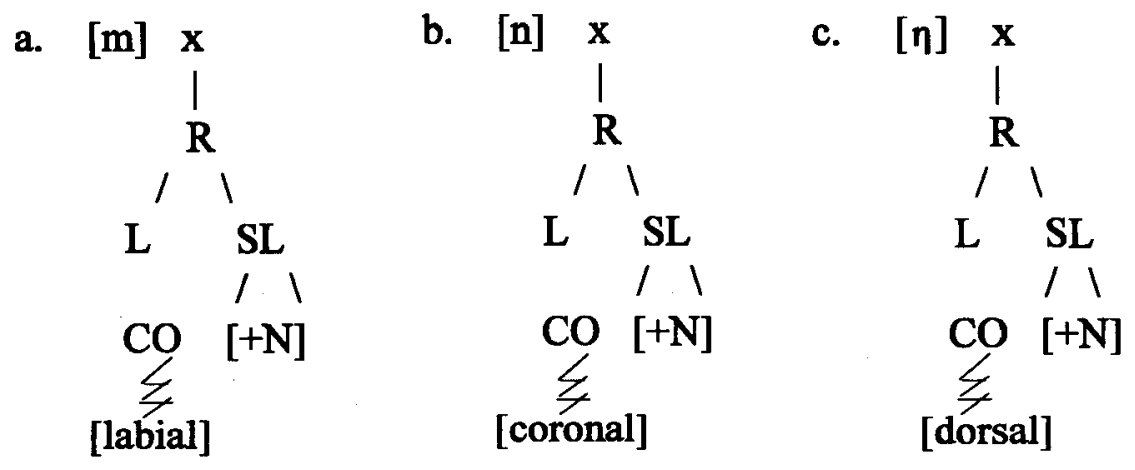

As representações acima mostram que o traço [+nasal] (doravante, $[+N]$ ) e o traço de cavidade oral(CO) de cada segmento, que ficam abaixo do nó raíz (R) e do nó supra-laringal (SL) de acordo com Clements (1991), estão especificados na representação subjacente.

Nessa língua, quando a raiz está especificada pelo traço [+cons], exceto nas oclusivas nasais, as obstruintes estão especificadas pelo traço [-nasal] (doravante, [-N]) e os traços da $\mathrm{CO}$, na representação subjacente, conforme mostra a representação (3): 
(3)

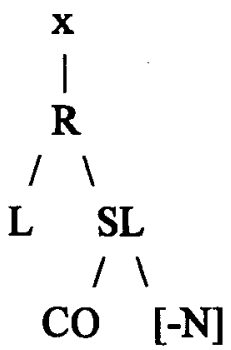

Assumimos que a nasalidade dos glides e das vogais não está especificada na representação subjacente, como demonstra (4a) e que, se o traço [nasal] ficar não especificado através da derivação, as vogais e os glides podem ser especificados [-N] redundantemente, como se mostra em (4b).

(4)

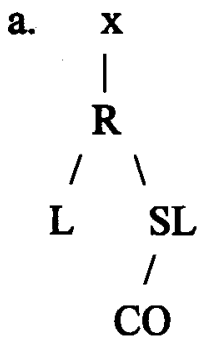

b. $\quad[] \rightarrow[-\mathrm{N}]$

A representação (4a) significa que, nas vogais e nos glides, somente os traços de CO estão especificados na representação subjacente.

\section{REGRAS FONOLÓGICAS DE NASAL NÃO- ESPECIFICADA}

3.1 Representação subjacente de nasal não-especificada

Considerem-se os seguintes exemplos: 
a.

sũwี 'pescoço'

ỹã̃̃a 'rato'

ũũ-t 'esconder alguma coisa'

ũhũk 'tosse'

kâi 'irmã'

wakt 'baixada'

hãã-t 'romper' b.

súwi 'escuro'

yáya 'estrela'

úut 'nome de aldeia'

uhúk 'rabo'

kái 'abacate'

wáki 'estômago'

haá-ču 'saro'

Os exemplos de (5) mostram que a nasalidade de vogais e de glides é a marca distintiva entre (5a) e (5b). De acordo com Payne (1974) e Corbera (1980), no entanto, essas nasalidades de vogal e glide não se encontram na representação subjacente; não existem os segmentos nasalizados na representação subjacente dessa língua. Em outras palavras, não há vogal nasal na representação subjacente do Aguaruna - a nasalidade de (5b) é derivada pela nasal não-especificada na representação subjacente, como mostra (6):

(6)
a. /yayaN/ $\rightarrow$

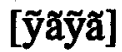
b. $/$ kaiN $/ \rightarrow$ [kâi $]$

Os exemplos de (6) mostram que a nasal não-especificada que fica no final da palavra nasaliza as vogais e os glides. Esse assunto será retomado na seção 3.2.3.

As consoantes nasais especificadas por $\mathrm{CO}$, diferentemente de nasal não-especificada, não são gatilhos para o processo de nasalização, como demonstram os seguintes exemplos:
a. ainc 'povo'
b. Cajkin 'cesta'
c. haanc 'tela'
d. datim 'medicina' 
Além disso, um processo de assimilação, abaixo exemplificada, favorece a presença de nasal não-especificado nessa língua.

(8)
a. /yayaN+ki/
yayanki 'somente rato'

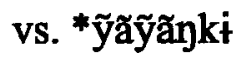
b. /piyuN+ču+i/
piyuncui 'não é inseto'
vs. *pîur̃nčui
c. /nuwaN+ču+i/
nuwančui 'não é mulher'
vs. *ñ̄ũwãnčui

Os exemplos de (8) mostram que, quando a nasal não-especificada (marcada por $\mathrm{N}$ nos exemplos) se encontra na posição precedida de oclusivas surdas na forma derivada, assimila os traços de C-place da oclusiva e, neste caso, a harmonização nasal para as vogais e os glides não acontece. ${ }^{4}$

Portanto, assume-se, neste trabalho, adotanto as idéias de Payne (1974) e Corbera (1980), que há um segmento especificado somente $[+N]$ na forma subjacente, gerado na posição Coda da sílaba ${ }^{5}$ que, ao se desligar, torna-se flutuante, e depois se espraia para a vogal e o glide (este assunto será discutido na seção seguinte), como em (9):

(9) a. forma gerada na base

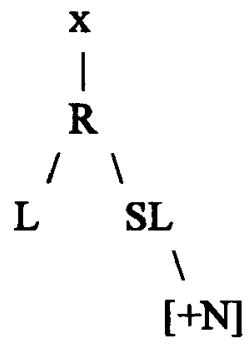

b. forma flutuante

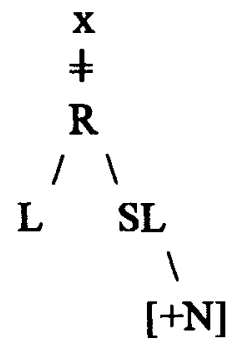

Neste trabalho, embora seguindo o modelo de Clements (1991), usaremos as representaçðes de Goldsmith (1976) para simplificar a explicação; isto é, (9) pode ser reescrito como (10): 

(10) a. $x$
b. $\mathbf{x}$<smiles>C[Al]=N</smiles>
$\neq$
$[+\mathrm{N}]$

As representações de (10) significam que o traço $[+\mathrm{N}]$ fica no nível auto-segmental; assim sendo, os traços de $\mathrm{CO}$ também podem ficar num nivel auto-segmental separado, (cf. Clements, 1985, 1989, 1991), como em (11):

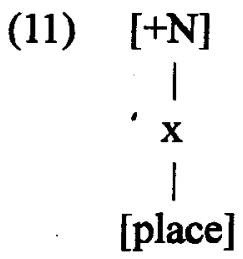

\subsection{Harmonização nasal}

\subsubsection{Harmonização de nasal flutuante}

Como mostramos rapidamente na seção anterior, a harmonização nasal do Aguaruna acontece com a nasal não-especificada na posição da Coda. Para explicar o processo de harmonização é preciso introduzir a noção de WFC, originalmente proposta por Goldsmith (1976) e depois reformulada por Halle \& Vergnaud (1981), como em (12):

(12) WFC

a. Cada slot é ligado a, pelo menos, um auto-segmento.

b. Auto-segmentos flutuantes são automaticamente ligados a todos os slots vocálicos acessíveis.

c. Auto-segmentos não-ligados são apagados no final da derivação.

d. Linhas de Associação não podem se cruzar.

Esta WFC prediz que a representação da harmonização nasal 
de (13a) é boa, satisfazendo as condições de (12), enquanto as representaçð̃es de (13b), não:

a. $\mathbf{X} \times \mathbf{x}$<smiles>[AlH2]</smiles><smiles>[X]C([X])C([X])[X]</smiles>

b. ${ }^{*} \mathrm{x} \quad \underset{\mathrm{I}}{\mathrm{x}} \mathrm{x}$ $[+\mathrm{N}]$<smiles></smiles><smiles>[X]C([Y])[AlH]</smiles><smiles></smiles>

$[+\mathrm{N}]$

À primeira vista, a harmonização nasal do Aguaruna está sujeita à WFC (12), como demostram as representações de (14):

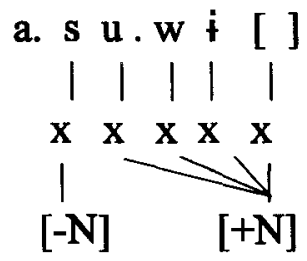

b. y a. y a [ ]

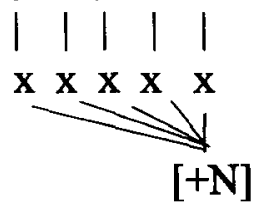

As representações acima mostram que a nasal não-especificada, gerada em posição da Coda, desliga-se do $x$ (slot) e torna-se uma nasal flutuante e depois se espraia para as Unidades Portadoras de $\mathrm{N}$ (N-bearing Units), cuja nasalidade não está especificada na representação subjacente. Quando essa nasal se torna flutuante, o x é apagado pela regra x-apagamento (Moon, 1988:127), como em (15):

(15)

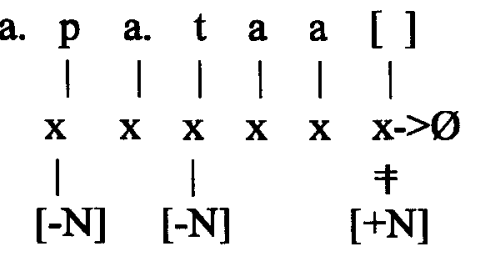

[patãã] 'família'

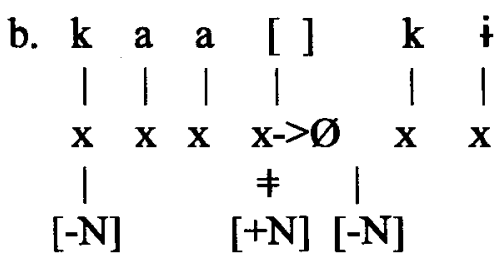

[kããkł] 'espécie de flor' 
De acordo com Clements \& Sezer (1982) e Piggott (1992), no Aguaruna, todas as obstruintes, inclusive as oclusivas nasais, bloqueiam o espraiamento de $[+\mathrm{N}]$, ou seja, funcionam como segmentos opacos ${ }^{6}$. Isso é o que realmente ocorre nos exemplos de (15), nos quais as obstruintes bloqueiam o espraiamento do traço [ $+\mathrm{N}]$, porque estão marcadas [-N] na representação subjacente. Se esse traço [+N] se espraiasse através de [-N], a condição (12d) da WFC não seria satisfeita, do que resultaria a forma: * pãtãã.

No entanto, Payne (1974:18) observa que a palavra/tuwiN + yaN/

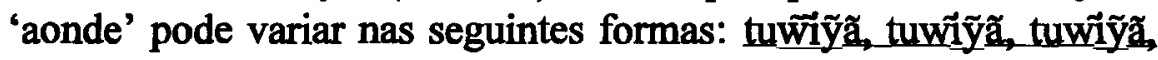

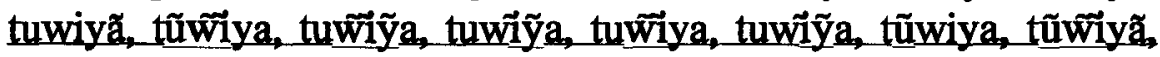
tũwิiỹa, tuwĩya, tũwīiyă, tũwiyã. Essas formas mostram que a WFC formulada em (12) não se mostra evidente no Aguaruna - a nasal nãoespecificada não se espraia para todas as unidades portadora de $\mathrm{N}$, embora as vogais e os glides sejam unidades de portadora de N. As representações de algumas dessas formas podem ser vistas em (16):

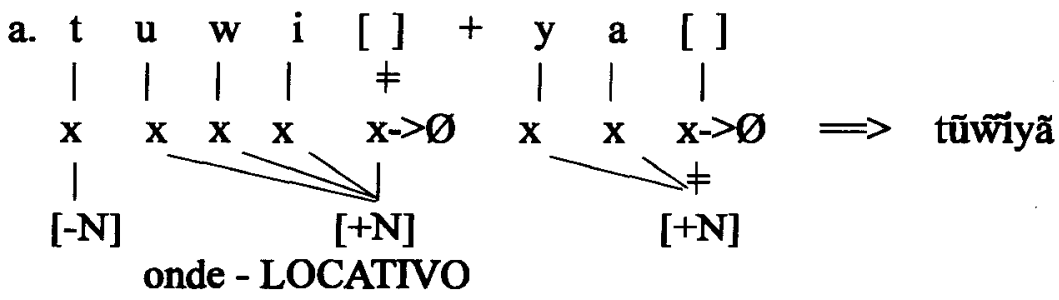

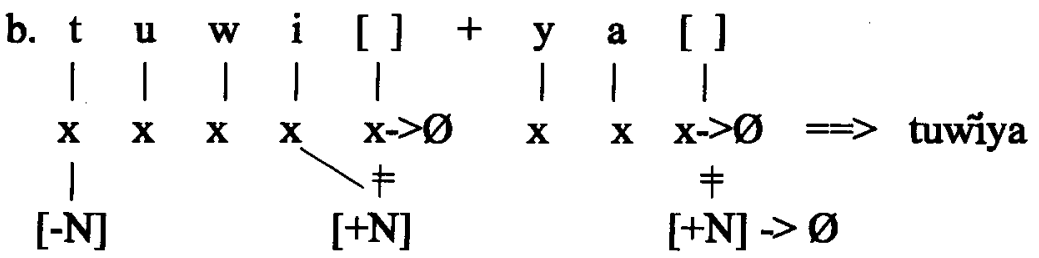

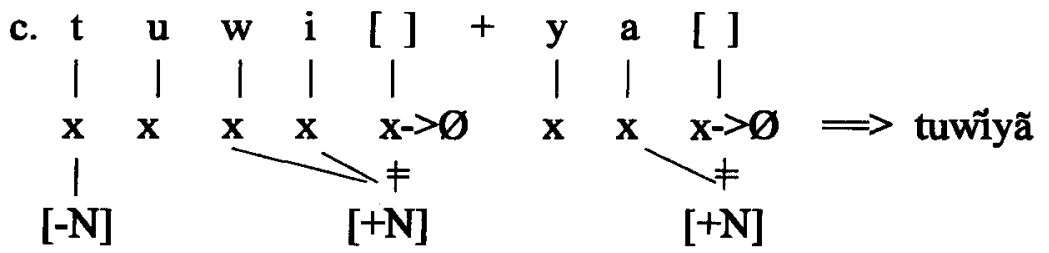


Em (16a), as nasais flutuantes se espraiam para as vogais e os glides, que não são marcadas pelo traço [nasal] na representação subjacente, de modo que a WFC é satisfeita;

Em (16b), no entanto, a primeira nasal flutuante se espraia somente para a vogal /i/, contrariando a WFC, e isso acontece também em (16c), a primeira nasal flutuante se espraia para a vogal /i/ e para o glide /w/, e a segunda nasal flutuante se espraia para a vogal /a/. ${ }^{7}$

Segundo Piggott (1992), considerando a distinção entre segmentos-alvo, segmentos opacos e segmentos transparentes ${ }^{8}$, o processo de harmonização nasal está sujeito a dois paradigmas possíveis:

I - Há um conjunto de consoantes que bloqueia o processo de nasalização e esse conjunto tem que incluir as obstruintes não-contínuas, como acontece, por exemplo, em Capanahua, Warao, etc.- o traço [nasal] depende do nó Soft Palate (SP);

II - Todas as obstruintes são transparentes e todas as soantes são alvos do processo, isto é, não há segmentos opacos para bloquear o processo, como se verifica, por exemplo, na maioria das línguas indígenas da América Latina, inclusive o Tupi e o Tucano - o traço [nasal] depende do nó Spontaneous Voicing (SV).

De acordo com esses dois paradigmas, a harmonização nasal resulta de dois fatos, respectivamente:

i) a harmonização é transmitida pelo espraiamento do nó SP, que domina o traço [nasal];

ii) o traço [nasal] se espraia para o nó SV, do qual depende.

Nas línguas incluídas no paradigma I, há segmentos-alvo e segmentos opacos, e a harmonização nasal tem como gatilho as consoantes nasais; nas línguas incluídas no paradigma II, há segmentosalvo e segmentos transparentes, e a harmonização nasal tem como gatilho a nasal flutuante gerada na base.

De um lado, o Aguaruna não pode ser enquadrado entre as línguas do paradigma II acima, pois nessa língua há segmentos opacos e não há segmentos transparentes. De outro lado, parece que o Aguaruna pertence ao paradigma I postulado por Piggott (1992), uma vez que, nessa língua: i) as vogais e os glides são segmentos-alvos, como em (14); ii) as obstruintes são segmentos opacos, como mostrou (15); 
iii) não há segmentos transparentes.

Entretanto, para esse paradigma I, o gatilho da harmonização nasal são as consoantes nasais da representação subjacente e, como mostramos na seção 3.1, no Aguaruna, as consoantes nasais não provocam o processo da harmonização nasal. ${ }^{9}$ Além disso, de acordo com o modelo de Piggott, nos exemplos (16b, c), o nó SP, que domina o traço [N], deveria se espraiar para todas as vogais e glides; isso, entretanto, não acontece, o que significa que esses exemplos não podem, portanto, ser explicados, à luz desse modelo. Realmente, nesses exemplos, $\mathrm{o}$ traço [ $\mathrm{+N}]$ não se espraia para todas as vogais e para todos os glides,

Com base em ( $16 \mathrm{~b}, \mathrm{c})$, pode-se perguntar, então:

i) O traço [nasal] do Aguaruna depende do nó Soft Palate?;

ii) Todas as vogais e todos os glides são segmentos-alvo no Aguaruna?;

iii) Se todas as vogais e todos os glides são segmentos-alvo no Aguaruna, o espraiamento do traço [ $+\mathrm{N}]$ é bloqueado em algum contexto?

Em primeiro lugar, considerando os exemplos, tais como a_nc, haan, verifica-se que, no Aguaruna, as consoantes nasais da representação subjacente não são gatilho da harmonização nasal e, observando as derivaçðes (15) verifica-se que as obstruintes são segmentos opacos. Esses fatos prevêem que a harmonização do traço $[+\mathrm{N}]$ do Aguaruna não pode depender de algum nó determinado, como pretende Piggott (1992). Isso é evidenciado também nos exemplos (16b, c), nos quais o espraiamento do traço [+N] não depende do nó Soft Palate nem do nó Spontaneous Voicing.

Em segundo lugar, como se observou na seção 2, as vogais e os glides do Aguaruna, desde que não especificados em [N] na representação subjacente, constituem segmentos-alvo "potenciais", ou seja, são segmentos que podem receber o traço [N] de um auto-segmento $[+\mathrm{N}]$. Acontece, no entanto, que nem todo segmento-alvo "potencial" recebe, necessariamente, o traço $[\mathrm{N}]$; em outras palavras, nem todo segmento-alvo "potencial" constitui um segmento-alvo "real". Se é assim, resta saber apenas sob que condições um segmento-alvo * 
potencial deve receber traço [N], ou não, o que será explicitado mais adiante.

Em resumo, os exemplos $(16 \mathrm{~b}, \mathrm{c})$ evidenciam que, de um lado, 0 Aguaruna não pode ser enquadrado entre as línguas do paradigma I, uma vez que: i) possui segmentos opacos e segmentos-alvo, mas nem todos os segmentos-alvo "potenciais" constituem segmentos-alvo "reais"; ii) as consoantes nasais não são gatilho para a harmonização nasal. Assim sendo, a teoria de Piggott (1992) não pode ser mantida para análise da harmonização nasal do Aguaruna, que deve ser explicada de outra maneira.

Retomando a noção de WFC (12), observa-se que as derivações $(16 \mathrm{~b}, \mathrm{c})$ violam as condições de WFC $(12 \mathrm{a}, \mathrm{b})$, ou seja, os segmentos cujas nasalidades estão não-especificadas na forma subjacente não estão associados a nasal flutuante na derivação. Pulleyblank (1986) argumentou que: essa noção da WFC é tão forte na análise do tom que, para explicar as associações múltiplas de um tom a mais de uma Unidade Portadora de Tom (Tone-bearing Unit) é preciso restringir a WFC e postular que tais associações ocorrem como o resultado de regras das línguas particulares. Adotando essas idéias de Pulleyblank (1986), assumimos que também em relação à harmonização nasal do Aguaruna faz-se necessário restrigir a noção de WFC. Em vista disso, propomos que (12) seja reescrita como (17):

(17) Condição de Não Cruzamento de Linha de Associação (CNLA) Linhas de associação não podem se cruzar.

Mas, essa CNLA também não é suficiente para explicar a harmonização nasal do Aguaruna. Essa CNLA vai gerar, nessa língua, muitas formas mal-formadas, tais como *yãyã, *yaya, *tũwĩya, *tuw̃iyã, *tuwiya, etc., embora esses exemplos satisfaçam a CNLA. Esse problema pode ser resolvido se se introduz uma Convenção de Associação do Aguaruna como (18): 
(18) Convenção de Associação do Aguaruna (CAA)

a. Somente segmentos de nasal flutuante são associados a, pelo menos, uma forte Unidade Portadora de $\mathrm{N}$.

b. As associaçres múltiplas de um $\mathrm{N}$ a mais de uma Unidade Portadora de $\mathrm{N}$ só é possível se essas Unidades Portadoras estão numa relação de adjacência.

c. Os auto-segmentos não-ligados são apagados no fim da derivação, se existir mais de um auto-segmento no domínio ${ }^{10}$.

A condição (18a) prediz que os glides não podem ser especificados, sozinhos, pela nasal flutuante, tais como *[yaya], *[tuwiya], etc. Quando eles se nasalizam, essa harmonização nasal deve ser realizada em, pelo menos, uma vogal, como em (19):

(19)

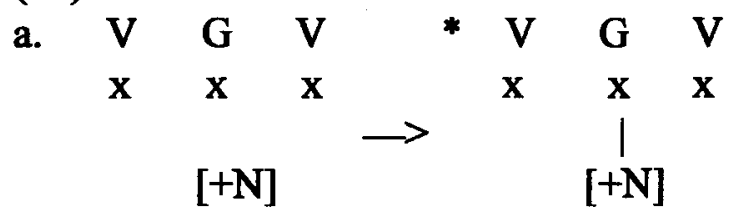

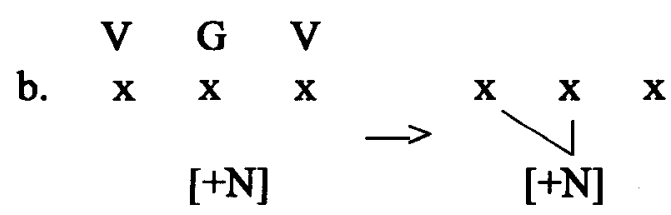

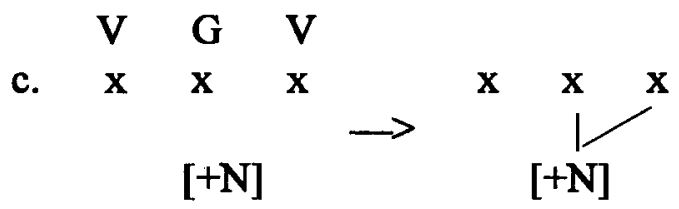

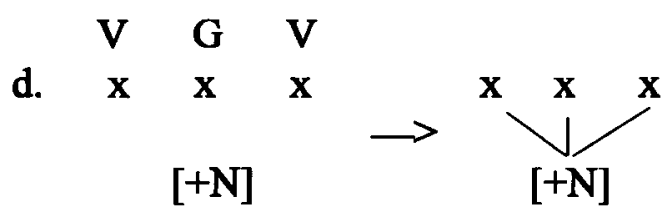


A condição (18b) prediz que associações múltiplas de um $\mathrm{N}$ a mais de uma Unidade Portadora de $\mathrm{N}$ só são possíveis se essas Unidades Portadoras estiverem numa relação de adjacência, como mostram as derivaçбes (14'), obtidas a partir de (14):
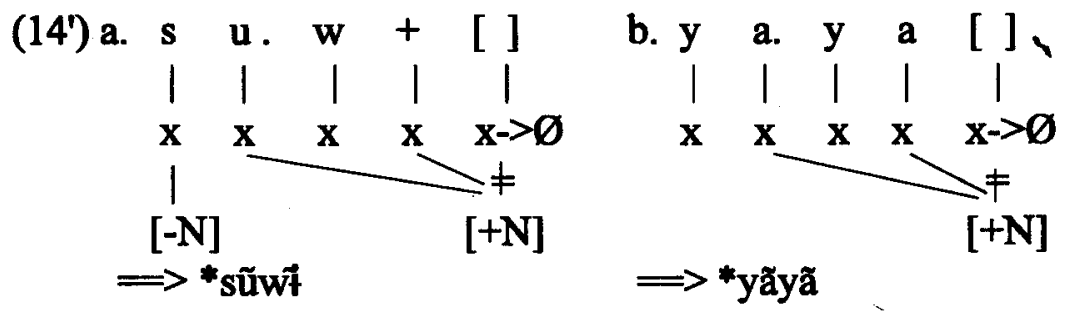

A condição (18c) prediz que os auto-segmentos não-ligados são apagados no final da derivação, como se mostra em (20):

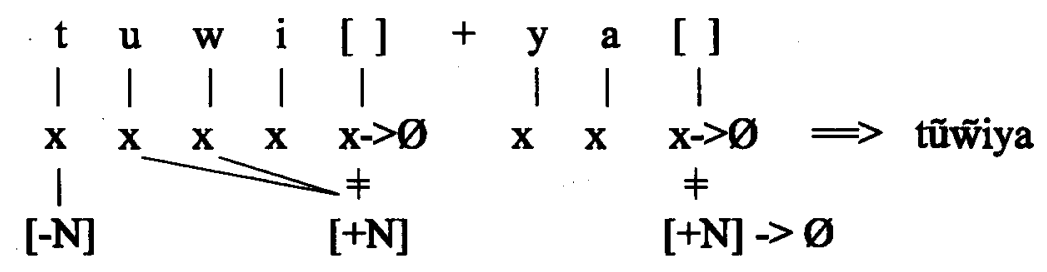

$\mathrm{Na}$ derivação de (20), observa-se que: a segunda nasal flutuante não se espraia para nenhum segmento - se a segunda nasal se espraia para / $u, w /$, isso viola a CNLA - e, consequentemente, é apagada no final da derivação; a primeira nasal flutuante se espraia para a vogal / u/ e 0 glide / $w /$. Mas o apagamento de nasal não-especificado não ocorre, quando há só uma nasal flutuante na representação no domínio: cf./yayaN/ -> *[yaya].

Retomando, então, as associações representadas em (13), é possível dizer que:

i) a associação de (13a) representa uma forma possível (bemformada) por satisfazer a WFC;

ii) as associaçōes de (13b), representam formas não-possíveis (mal-formadas), por não satisfazerem a WFC.

De acordo com a CNLA e a CAA para a análise da harmonização 
nasal do Aguaruna, além da associação (13a), também associações do tipo que se encontra em (13b) representam formas possíveis (bemformadas), uma vez que satisfazem a CNLA e a CAA. Considerando tal fato, essas associaçð̃es serão reescritas como (13'):

(13')

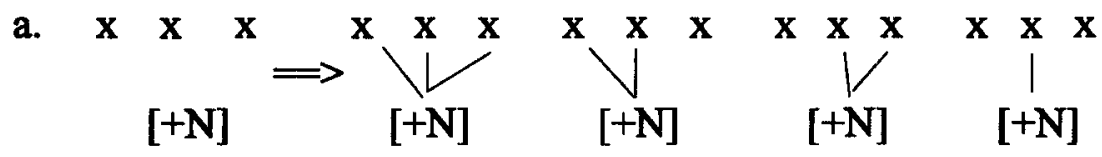

b. ${ }^{*} \mathrm{x}{ }^{\mathrm{x}}$

$[+\mathrm{N}]$

Retomando, mais uma vez, os exemplos $(16 \mathrm{~b}, \mathrm{c})$, eles podem ser reescritos como em (16'b, c):

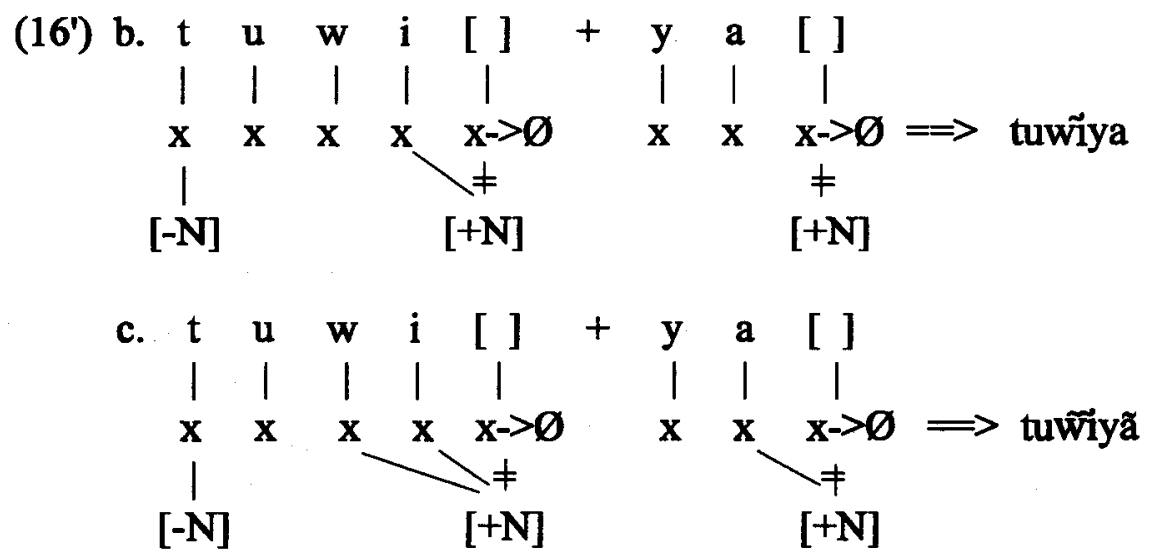

Nas derivaç̃es de $\left(16^{\prime}\right)$, observa-se que: em (16'b), a primeira nasal flutuante se espraia somente para a vogal $/ \mathrm{i} /$, satisfazendo a CNLA e a CAA; em (16'c), caso em que a primeira nasal flutuante se espraia para a vogal $/ \mathrm{i} /$ e para o glide $/ \mathrm{w} /$, e a segunda nasal flutuante se espraia para a vogal /a/, também são satisfeitas a CNLA e a CAA. Em síntese, postulando-se a CNLA e a CAA, é possível explicar a 
harmonização nasal do Aguaruna naqueles casos em que esse fenômeno não é explicado pela WFC nem pela teoria de Piggott (1992).

\subsubsection{Direcionalidade da Harmonização nasal}

Nos estudos de Fonologia Auto-segmental, a direção de espraiamento dos traços também ocupa uma posição importante. Assumese, portanto, que no Aguaruna o traço $[\mathrm{N}]$ pode espraiar para ambos os lados dentro do domínio, uma vez que não viola a CNLA e a CAA, como mostra (21):

$$
\begin{aligned}
& \text { a. } \quad \begin{array}{lllllll} 
& \mathbf{1} & \mathbf{w} & \mathbf{a} & \mathbf{n} & \tilde{\mathbf{c}} & \mathbf{i}
\end{array}
\end{aligned}
$$

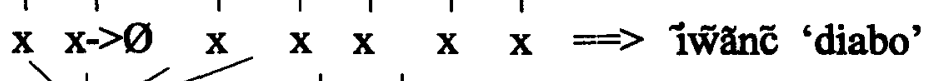

$$
\begin{aligned}
& {[+\mathrm{N}] \quad[+\mathrm{N}][-\mathrm{N}]} \\
& \text { b. } \begin{array}{cccccccc}
n & \mathbf{i} & \mathbf{h} & \mathrm{a} & \mathrm{y} & +\mathrm{i} & {[\mathrm{l}}
\end{array}
\end{aligned}
$$

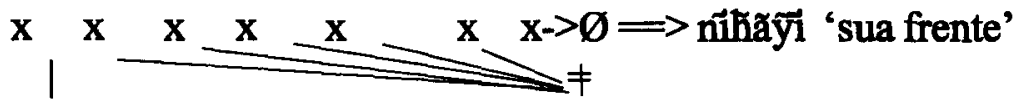

$$
\begin{aligned}
& {[+\mathrm{N}] \quad[+\mathrm{N}]}
\end{aligned}
$$

Os exemplos de (21) satisfazem a CNLA e a CAA, (21c), por exemplo, pode variar realizando-se das seguintes formas: ayawai, ayawăi, ayãwãi, etc. 


\subsubsection{Assimilação de Nasal Não-Especifícada}

Como se observou na seção 3.1, há processo de assimilação de nasal não-especificada quando essa nasal se encontra no ambiente derivado; ou seja, na posição seguida por obstruintes não-contínuas, os traços de C-place de nasal não-especificada assimilam os traços de C-place das obstruintes seguintes, na forma derivada (Kiparsky (1982)), ${ }^{11}$ como em (22), em que não ocorre a harmonização de nasal:

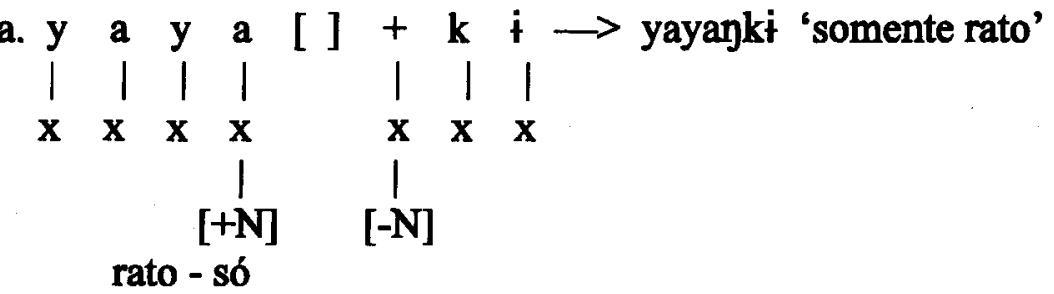

b. $n$ i i [ ] + p a $\quad$ s $\rightarrow$ niimpas 'ele/ela?'

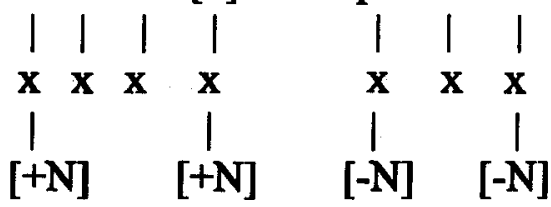

ele/ela - INTERROGATIVO

c. $k$ a $n$ u + h a i [ ] + č $u \rightarrow$ kanuhainču 'não com canoa'

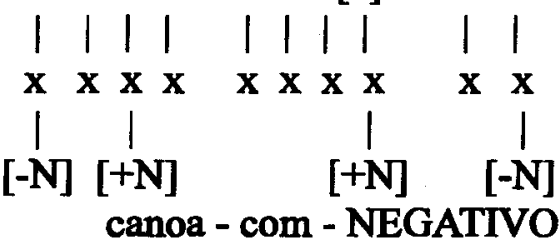

d. $\mathbf{d}$ a s $\mathbf{i}$ [ ] $+\mathbf{a}+\mathbf{k} \dot{\mathbf{i}} \longrightarrow$ dasłåki 'seguramente

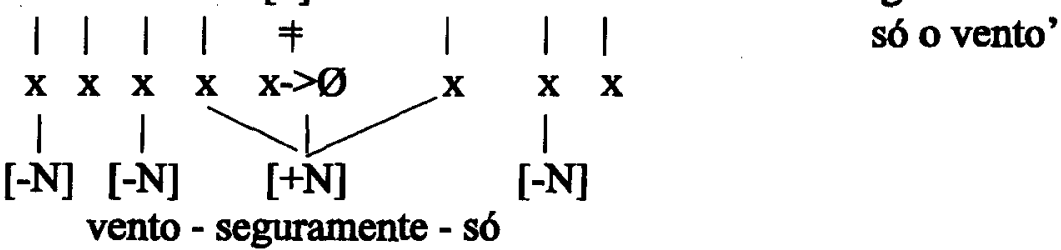




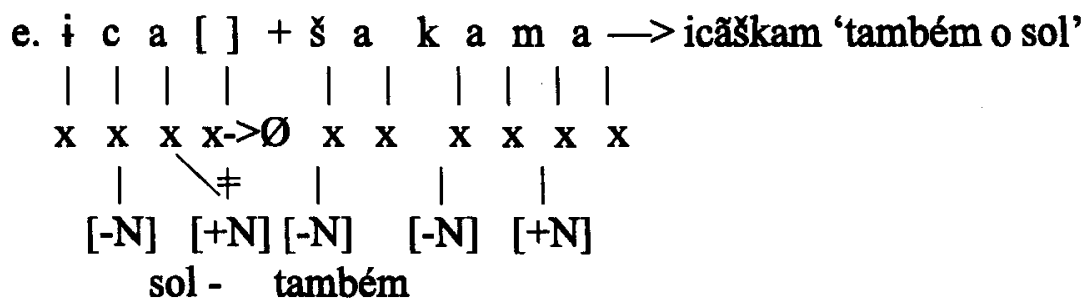

Qs exemplos de (22a-c) mostram que cada nasal não-especificada assimila o traço de C-place das obstruintes seguintes; no entanto, em (22d), a nasal não-especificada, gerada na posição final do primeiro morfema, não se encontra diretamente do lado da obstruinte, isto é, o segundo morfema, /a/ intervém entre a nasal não-especificada e a oclusiva, de tal modo que o processo de harmonização nasal ocorre em lugar do processo de assimilação; (22e) mostra que, com a obstruinte contínua, não ocorre a assimilação, mas ocorre o processo de harmonização nasal. Este fato, portanto, pode ser assim formalizado:

(23) Assimilação Nasal ${ }^{12}$

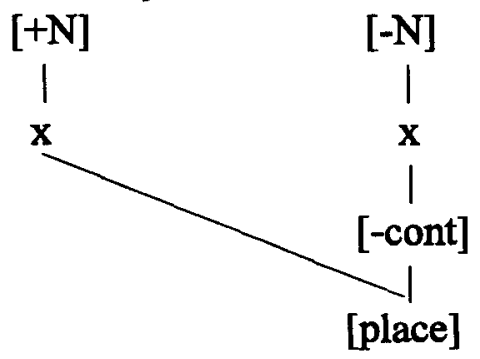

\subsection{Desnasalização}

No Aguaruna, existe variação livre entre $/ \mathrm{m} / \mathrm{e} / \mathrm{b} / \mathrm{e}$ entre $/ \mathrm{n} / \mathrm{e} / \mathrm{d} /$ , em determinados contextos, como demonstra (24): 
(24)

$\begin{array}{lll}\text { a. mwitik } & \text { bwitik } & \text { 'igual' } \\ \text { máku } & \text { báku } & \text { 'perna' } \\ \text { nihéi } & \text { dihéi } & \text { 'frente' } \\ \text { nítsi } & \text { dítsi } & \text { 'louco' }\end{array}$

b. nánčik *dánčik 'unhas'

$\begin{array}{lll}\text { nii } & \text { *dị } & \text { 'ele, ela' } \\ \text { mâi } & \text { *båi } & \text { 'ambos' } \\ \text { mwina } & \text { *bwina } & \text { 'esquerda' }\end{array}$

Os exemplos de (24a) mostram que se os sons especificados [ $+\mathrm{N}]$ na representação subjacente, que ficam na posição do Onset da sílaba, encontrarem uma vogal [ $-\mathrm{N}]$ mais um elemento [-N], seguidos na forma fonética, estas nasais podem perder o traço [+N], mas não perdem outros traços ${ }^{13}$; já em (24b), este processo de desnasalização não ocorre, porque as nasais na posição do Onset da sílaba ficam ao lado dos segmentos nasais e dos segmentos nasalizados. Este processo de desnasalização pode ser formalizado como em (25):

(25) Desnasalização

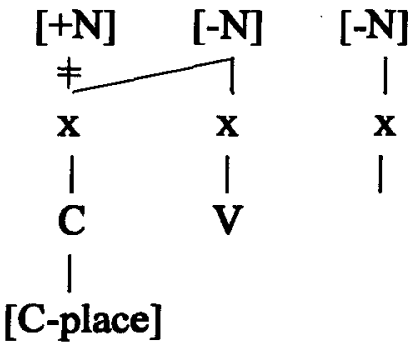

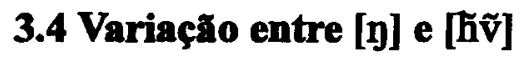

Há um processo de harmonização nasal entre os processos derivacionais do Aguaruna - o morfema $/ \mathrm{gu} /$, que manifesta aspecto verbal ou possessivo, pode ocorrer como [hũ] ou [n], dependendo do contexto, como em (26): 
(26)

a. uči -n

filho-POSS 'meu filho'

b. buuk+ - n cabeça-POSS

'minha cabeça'

c. duha - $\mathrm{n}$ - tin

o rio aumentar-ASP-FUTURO

'o rio aumentará'
a'. uči - h̆̃̃ - n
filho-POSS-ACUS
'meu filho'
b'. buuk - hũ - ka
cabeça-POSS-INTER
'minha cabeça?'
c'. duha - Kũ - $t$
o rio aumentar-ASP-INF
'o rio aumentar'

Essa nasal velar nunca aparece na posição do Onset da sílaba na forma de superfície, diferentemente de [n] e [m]. Esta nasalidade é gatilho para um processo de nasalização, mas, nesse caso, o domínio da harmonização nasal é só a sílaba, ou seja, não se nasaliza fora da sílaba, enquanto o domínio da harmonização nasal da nasal flutuante é a palavra, como mostramos na seção 3.2.2.

Os exemplos de (27) mostram que essa variação entre [hã] e [y] também ocorre dentro da forma primitiva, isto é, nos casos em que não há fronteira morfológica.

Nominativo

a. himan 'dois' caČn 'espécie de árvore' sapin 'medroso'

Declarativo

b. siin 'bem, bonito' pinkin 'bom, limpo'
Acusativo

himaగ̌ã-n

cačiłã-n

sapít็a-n

Interrogatvio

siiȟã-k

pł̣kłinã-k 
Como se pode observar, a forma nominativa [himan], derivada da representação subjacente /himana/, quando toma a forma acusa-

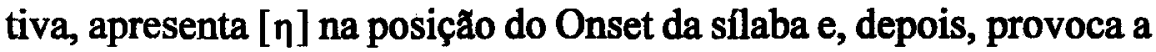
nasalização dentro da sílaba.

Este fato pode ser formalizado da seguinte forma:

(28) Nasalização de /y/

Coda

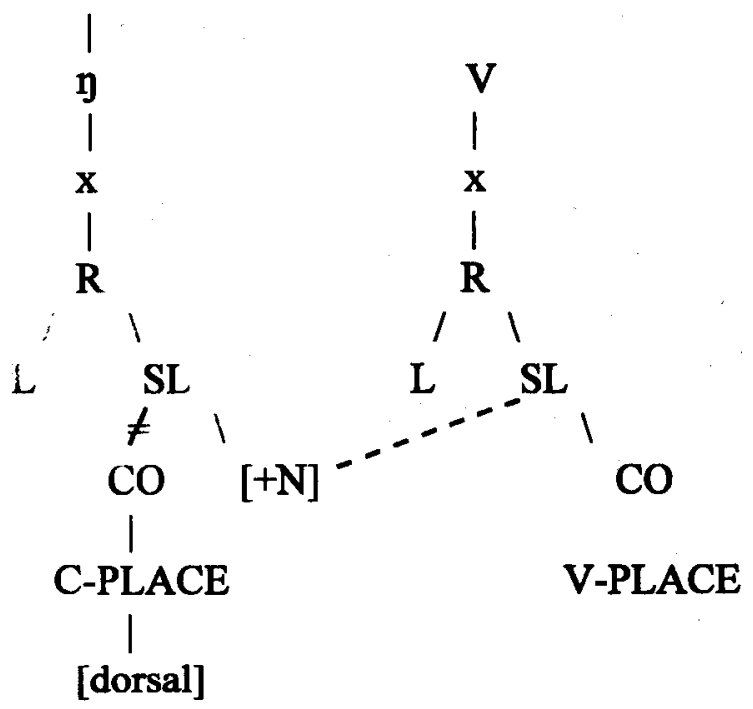

Esta regra prediz que, quando nasal velar fica na posição do Onset da sílaba, torna-se uma nasal "semi-flutuante" e nasaliza a vogal que a segue no domínio da sílaba. Esta noção "semi-flutuante" é introduzida para diferenciar da nasal não-especificada - que se torna a nasal flutuante na harmonização nasal.

Considerem-se os seguintes exemplos.

(29)
a. ãกัuีt
'coisa velha'
b. uีกũ ' 'algodâo'
c. akahũ
'espingarda'
d. huhif-t 'parir'
e. ãกัน์
'mais tarde'
f. sũกũ-t 'vender'
g. ãกã-t
'plantar
h. Sînũkap 'lume de borracha' 
Os exemplos de (29) mostram que a cada palavra possui a forma fonética [గĩ]. Então, é possível postular que os seguintes exemplos também são derivados da forma subjacente $/ \mathfrak{\eta V} /$ ? É difícil considerar esse [hṽ] como derivado de $/ \mathrm{gV} /$, porque, em primeiro lugar, esses

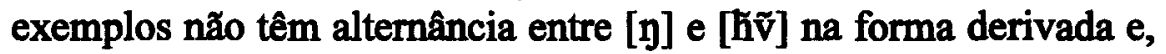
em segundo lugar, a harmonização nasal verificada nestes exemplos é idêntica à que ocorre nos casos de nasal não-especificada (cf. [ah̃̃̃t], [ahũt]), em que a CNLA e a CAA são satisfeitas, como mostrou a seção 3.2.1. Entretanto, os exemplos de (27) mostram que a harmonização nasal não pode atravessar a sílaba: *[himãhă], *[sưihăk]. Isso significa, portanto, a não existência de alternância entre [y] e [inv] nos exemplos de (29), em que $/ \mathrm{hV} /$ é forma primitiva, conforme ilustram as representações subjacentes de (29a, c), a seguir:

(29a') /ahuNt/

(29c') /akahuN/

Retomando os exemplos (26c, c'), é possível observar que, quando o morfema $/ \eta u /$ é precedido por nasal não-especificada, provoca o apagamento dessa nasal não-especificada. Este fenômeno pode ser formalizado através da regra (30) e ilustrado através do contraste entre (31a) e (31b, c):

(30) Apagamento de nasal não-especificada

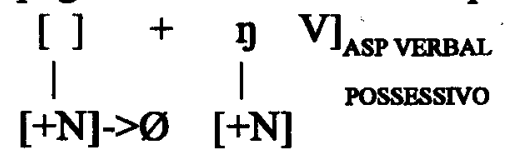

(31)

a. $\mathbf{n}$ u h a [ ] + w a $+\mathbf{i} \Longrightarrow$ nũћãwãâi 'O rio está aumentando'

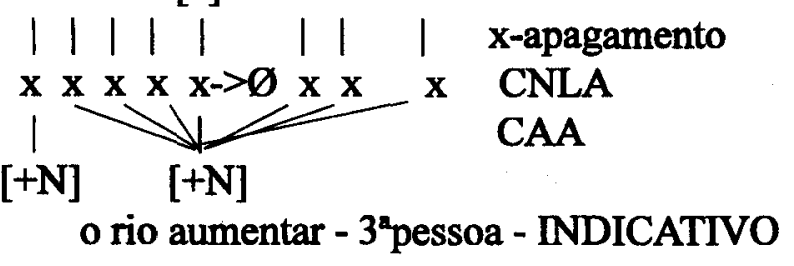




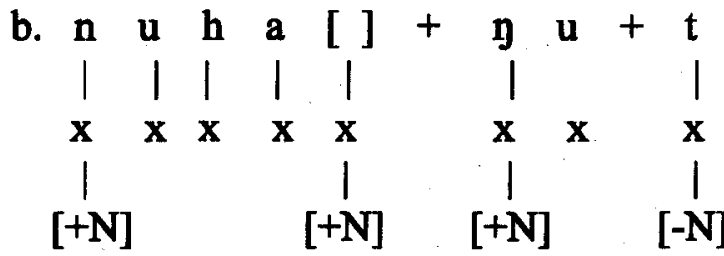

ASPECTO - INFINITIVO

$\Longrightarrow$ Regra (30)

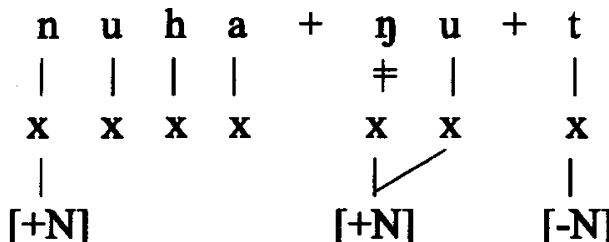

Regra (28) e (25)

$\Rightarrow \quad$ duhahũt

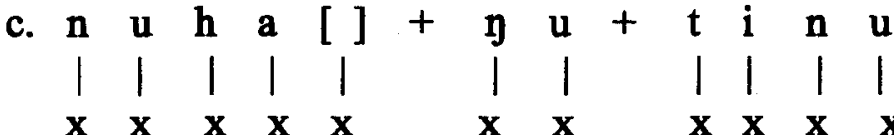

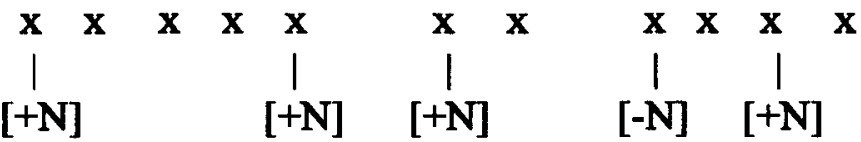

ASPECTO - FUTURO

$\Longrightarrow$ Regra (30) e Restrição de Sílaba

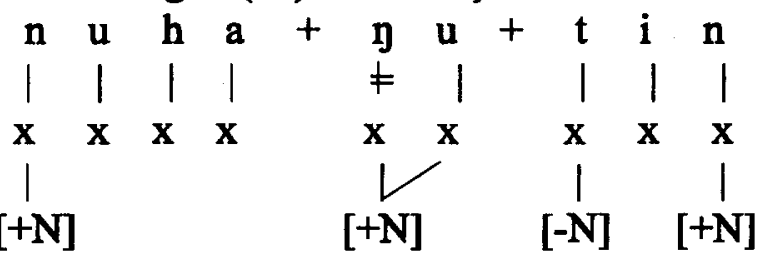

Regra (28) e (25)

$\Rightarrow$ duhahũtin

A derivação (31a) - que é a forma de $3^{a}$ pessoa do indicativo mostra que a nasal não-especificada se torna flutuante e, depois, es- 
praia seu traço para vogais e glides, satisfazendo a CNLA e a CAA. Em (31b), a nasal não-especificada cai em frente do morfema / $\mathrm{u/,}$ isto é, não provoca harmonização nasal e, depois, a regra (25) aplicase, conforme se disse. $O$ ponto mais importante neste exemplo é o seguinte: depois do apagamento da nasal não-especificada, a nasal velar, que fica na posição do Onset da sílaba, se torna "nasal semiflutuante", ou seja, perde seu traço de CO e nasaliza a vogal dentro da sílaba. Uma vez que esta harmonização nasal não atravessa a fronteira morfológica - *[dũhãhũt], *[nũhãhũt], etc. - (31c) é derivado da mesma maneira que (31b); além disso, a última vogal / $\mathrm{u} /$ cai como resultado da regra de restrição silábica da língua na derivação, conforme Corbera (1991).

\section{CONCLUSÃO}

Este trabalho, adotando a teoria da Geometria de Traços de Clements (1991), mostrou que o processo de harmonização nasal do Aguaruna é tratado como diferente de outros processos de harmonização - a harmonização de [ATR], a harmonização de [ \pm back] e a harmonização de tom - explicados, na literatura, como processos sujeitos somente a condições universais.

No Aguaruna, há três tipos de segmentos nasais, como se pode ver em (32): (32a) é a representação da nasal não-especificada que provoca a harmonização nasal; (32b) é a representação das oclusivas nasais; (32c) é a representação da nasal não-especificada que copia o traço de C-place do segmento seguinte na derivação.

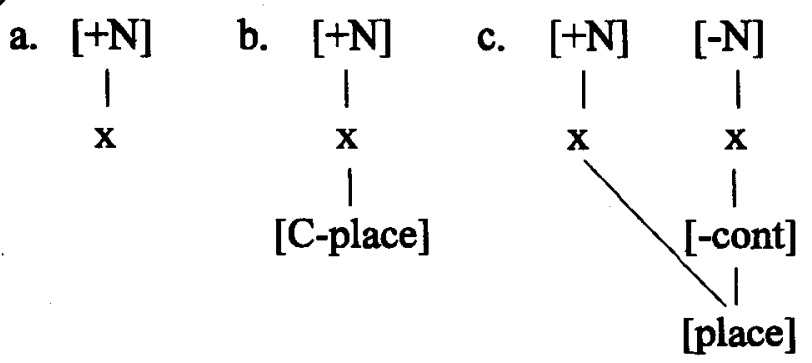


Em relação ao processo da harmonização nasal do Aguaruna, é possível afirmar que, nessa língua:

a) o gatilho da harmonização nasal é a nasal não-especificada e a nasal velar na posição Onset da sílaba;

b) há dois domínios de espraimento do [+N], ou seja, a nasal não-especificada provoca a harmonização nasal no domínio da palavra, enquanto a nasal velar na posição do Onset da sílaba provoca a harmonização nasal no domínio da sílaba;

c) existem segmentos-alvo e segmentos opacos, mas não existem segmentos transparentes;

d) os segmentos-alvo incluem as vogais e os glides, mas esses segmentos-alvo "potenciais" nem sempre constituem segmentos-alvo "reais", em outras palavras, as vogais e os glides são os segmentos que podem receber o espraiamento do traço $[+\mathrm{N}]$ de um auto-segmento, mas o traço [ $+\mathrm{N}]$ de um auto-segmento não precisa se espraiar para todas as vogias e para todos os glides.

e) todas as obstruintes (inclusive as oclusivas nasais) são segmentos opacos.

f) O processo de harmonização nasal deve satisfazer a CNLA e a $\mathrm{CAA}$, ou seja, o traço $[+\mathrm{N}]$ năo depende de um determinado nó.

\section{NOTAS}

*

Agradeço a Prof. Luiz Carlos Cagliari, Prof. Leo Wetzels, Profa. Maria Bernadete Abaurre, Profa. Leda Bisol, Profa. Lucy Seki, Prof. Marco Antônio de Oliveira, Prof. Angel Mori Corbera e parecerista anônimo (a) desta revista pela leitura com generosos comentários e valiosas sugestóes e a Eunice Nicolau pela correção do português. Todos os erros que persistem são da minha inteira responsabilidade.

1

Considero que o princípio de Espraiamento e o Princípio de Aplicação Máxima de Piggott são iguais a WFC (14a, b, d).

2

Os dados deste trabalho são de Corbera (1981) e Payne (1974). 
3

No sistema consonantal de Aguaruna, a consoante oclusiva velar sonora /g/ não 4 ocorre

Payne (1974) e Moon (1988) mostram que os falantes mais jovens dessa língua pronuciam tais formas, ou seja, essas palavras podem ser assim pronunciadas:

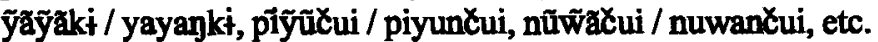

5

Assumiremos a seguinte estrutura silábica:

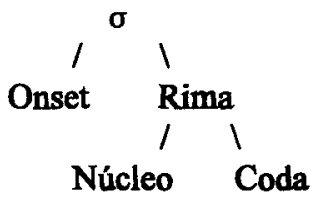

6

As condiçбes (12 a, b, c) são propostas por Halle \& Vergnaud (1981) e a condição (12d), por Goldsmith (1976).

7

Esta noção é adotada por Clements \& Sezer(1982), ou seja, $N$-Bearing Units significam as vogais e os glides. Também usaremos a noção de forte para distiguir as vogais dos glides.

8

Mas a fricativa velar sonoro $/ \gamma /$ não bloqueia o processo da harmonização nasal, como as vogais e os glides. Por isso, assumimos que esta fricativa também fica não especificada quanto à nasalidade na representação subjacente.

9

Nas outras formas de realização [tũพิ์ịã] verifica-se esse mesmo fato.

10

. Segmentos-alvo são os possíveis candidatos a receber o espraiamento do autosegmento; segmentos opacos são aqueles que se interpõem entre dois segmentosalvo, de modo que impedem o espraiamento do auto-segmento para esses dois segmentos-alvo ao mesmo tempo; segmentos transparentes são aqueles que se interpøem entre dois segmentos-alvo e permitem que o espraiamento do autosegmento atinja esses dois segmentos-alvo ao mesmo tempo.

11

No Aguaruna, as consoantes nasais $/ \mathrm{m} / \mathrm{e} / \mathrm{n} /$ não provocam a harmonização nasal, e a consoante nasal $/ \mathfrak{y} /$ só provoca a harmonização nasal quando está na posição do Onset da sílaba. cf. seção 3.4. 
12

A noção de domínio adotada aqui é de Hulst \& Smith (1982b) e Clements \& Sezer (1982), segundo a qual, no Aguaruna o espraiamento do traço [ $+N]$ de nasal flutuante se dá na palavra.

13

Com a Strict Cycle Condition (doravante, SCC) de Kiparsky (1982), podem-se explicar palavras como [șūw̃ãpa] 'mosca', [kãăk+] 'flor', derivadas das representaçð̄es subjacentes /suwaNpa/e / kaaNk $+/$ : como nesses casos não há ambiente de assimilação de nasal, não ocorre o processo de assimilação; isto significa que estas palavras são formas não-derivadas.

14

As análises de Payne (1974) e Moon (1988) mostram que essa assimilação ocorre nas formas não-derivadas. Essas análises, no entanto, não podem explicar por que as consoantes nasais não motivam o processo de harmonização nasal.

15

O fonema / $/ \mathbf{y}$ não aparece na posição Onset na forma derivada dessa língua.

\section{BIBLIOGRAFIA}

Clements, G. N. (1985) The Geometry of Phonological Features, Phonology Yearbook 2.

Clements, G. N. (1987a)Phonological Feature Representation and the Description of intrusive Stops, CLS 23, pp 29-50.

NELS 18, 79-93.

(1987b) Towards a Substantive Theory of Feature Specification,

Clements, G. N. (1989) A Unified Set of Features for Consonants and Vowel, Ms, Cornell University.

(1991) Place of Articulation in Consonants and Vowels: a Unified Theory, Working Papers of the Cornell phonetics Laboratory 5, 77123.

Clements, G. N. \& Sezer, E. (1982) Vowel and Consonant Harmony in Turkish, In Hulst \& Smith (eds) (1982a), Part II.

Corbera, Angel M. (1980)Algunos Processos Morfofonemicos que Subyacen en la Fonologia Aguaruna, em Amazonia Peruana Vol. IIINO 5, pp. 103-120. 
Rev. Est. Ling., Belo Horizonte, ano 4, v. 2, p. 197-256, jul./dez. 1995

(1981)Glosario Aquanuna-Castellano, Centro de Investigacion de Linguistica Aplicada, Universidad Nacional Mayor de San Marcos.

(1991) Fonologia de Regência: A Queda de Vogais no Aguanuna, manuscrito, Unicamp.

Cohn, A. C. (1990) Phonetic and Phonological Rules of Nasalizàtion, UCLA Working Papers in Phonetics 76.

Goldsmith, J. A. (1976) Autosegmental Phonology, Doctoral dissertation, MIT, Cambridge, Mass.

Halle, M.\& J, -R. Vergnaud (1981) Harmony Process, In Klein, W. \& W. Levelt (eds) Crossing the Boundary in Linguistics, Reidel, Dordrecht.

Halle, M.\& J, -R. Vergnaud (1982) On the Framework of Autosegmental Phonology, In Hulst \& Smith (eds.) (1982a) Part I.

Hulst, H. v. d. \& N. Smith, ed. (1982a) The Structure of Phonological Representations, Part I \& II, Foris.

Hulst, H. v. d. \& N. Smith, ed. (1982b) Prasodic Domain and Opaque Segments in Autosegmental Theory, In Hulst \& Smith (eds.) (1982a), Part II.

Hyman, L. M. (1981) The Representation of Nasality in Gokana, In Hulst \& Smith (1982a), Part I.

Kiparsky, P. (1982) Lexical Morphology and Phonology, in I.-S. Yang ed., Linguistics in the Morning Calm, Hanshin, Seoul, 3-91.

McCarthy, J. (1988) Feature Feometry and Dependency : A Review, Phonetica 43.3.

Moon, G.-S. (1988) Nasal Harmony in Aguanma, Texas Linguistic Forum 29, pp. 115-146.

Payne, (1974) Nasality in Aguaruna, M. A. Thesis, The University of Texas at Arlington (Traduzido para o Espanhol por María L. de Cerrón-Palomino, Serie Linguistica Peruana Publicacion Numero 13, 1976)

Piggott, G. L. (1987) On the Autonomy of the Feature Nasal, CLS 23, 223-238. 
Piggott, G. L. (1992) Variability in Feature Dependency: The Case of Nasality, Natural Language and Linguistic Theory, 32-77.

Pulleyblank, D. (1986) Tone in Lexical Phonology, Reidel, Dordrecht.

E-MAIL: SHLEE.@ORACULO.LCC.UFMG.BR 